\title{
Barrier Lyapunov Function-Based Adaptive Back-Stepping Control for Electronic Throttle Control System
}

\author{
Dapeng Wang ${ }^{1,2, *(\mathbb{D}}$, Shaogang Liu ${ }^{1}\left(\mathbb{D}\right.$, Youguo $^{3} \mathrm{He}^{3}$ and Jie Shen ${ }^{4}(\mathbb{D}$ \\ 1 College of Mechanical and Electrical Engineering, Harbin Engineering University, Harbin 150001, China; \\ liu_shaogang@hotmail.com \\ 2713 Research Institute of China Shipbuilding Industry Corporation, Zhengzhou 450015, China \\ 3 Automotive Engineering Research Institute, Jiangsu University, Zhenjiang 212013, China; \\ hyg197715@163.com \\ 4 Department of Computer and Information Science, University of Michigan-Dearborn, \\ Dearborn, MI 48128, USA; shen@umich.edu \\ * Correspondence: wdp_heu@163.com
}

Citation: Wang, D.; Liu, S.; He, Y.;

Shen, J. Barrier Lyapunov

Function-Based Adaptive

Back-Stepping Control for Electronic

Throttle Control System. Mathematics

2021, 9, 326. https://doi.org/

$10.3390 /$ math 9040326

Academic Editor: Temirkhan Aleroev

Received: 23 December 2020

Accepted: 31 January 2021

Published: 6 February 2021

Publisher's Note: MDPI stays neutral with regard to jurisdictional claims in published maps and institutional affiliations.

\begin{abstract}
This paper presents an adaptive constraint control approach for Electronic Throttle Control System (ETCS) with asymmetric throttle angle constraints. The adaptive constraint control method, which is based on barrier Lyapunov function (BLF), is designed not only to track the desired throttle angle but also to guarantee no violation on the throttle angle constraints. An ETC mathematic model with complex non-linear system is considered and the asymmetric barrier Lyapunov function (ABLF) is introduced into the design of the controller. Based on Lyapunov stability theory, it can be concluded that the proposed controller can guarantee the stability of the whole system and uniformly converge the state error to track the desired throttle angle. The results of simulations show that the proposed controller can ensure that there is no violation on the throttle angle constraints.
\end{abstract}

Keywords: electronic throttle control; constraint control; barrier Lyapunov function; throttle opening angle; adaptive back-stepping control

\section{Introduction}

The electronic throttle control system (ETCS) is one of the main engine electronic control system (ECU) devices, which has a function to control the air intake of the engine cylinder by adjusting the position of the electronic throttle valve accurately. Therefore, the research of ETCS control algorithm has attracted extensive attention in the past few years.

Previously, the ETCS control algorithm usually used proportional-integral-derivative (PID) control to calculate the required throttle opening angle [1]. In [1], a control strategy of automotive ETCS based on PID control is proposed. However, this method relies on empirical or experimental adjustment parameters to a large extent, and it is easy to overshoot, affecting the stability. Therefore, improving the control performance of ETS based on throttle angle control algorithm has become a research focus in recent years. Such as sliding mode control methods (SMC) [2-4], backstepping control [5-8], fuzzy/neural network controls [9,10], and other control methods.

In [2], according to the variation of the angle of the electronic throttle (ET), a new extended state observer method is proposed, which takes into account the stick-slip friction, the nonlinear uncertainty of the spring, and the external disturbance of the system. In [3], for the robustness of the electronic throttle valve (ETV) system, a robust controller is designed based on the theory of integral sliding mode control. An adaptive sliding-mode tracking control strategy for an ET is applied in [4]. In this approach, input saturation constraints and parameter uncertainties are fully considered, and a parameter adaptive law is proposed to estimate unknown parameters with ET. An auxiliary system is designed to handle the input saturation. In [5], a finite-time convergence chattering-free backstepping 
sliding mode controller of electronic throttle was designed. Nonlinear disturbance observer and continuous high gain method were used to eliminate chattering. To improve the tracking performance of the electronic throttle, in work by Chen $\mathrm{H}$ et al. [6], a controloriented nonlinear electronic throttle model is proposed. The input shaping technique is applied to the filtering of the input signal, and the nonlinear controller is obtained by using the backstepping technique. In $[7,8]$ Nobuo Kurihara et al. proposed backstepping control (BSC) to improve both response speed and resolution performance of the engine's electronic control throttle (ECT). In [9], aiming at the nonlinear hysteresis characteristic of electronic throttle, an intelligent fuzzy feedback control strategy with feedforward compensation is proposed. However, the expected effect of the fuzzy rule to compensate for the nonlinear hysteresis of the ET is not ideal. In [10], two control technologies are adopted to control the speed of the given two kinds of heavy-duty vehicles with different random inputs, which are called the standard PID controller and the adaptive neural network fuzzy reasoning controller, respectively. By comparison, the latter method has better performance. In [11], considering that there are a lot of non-smooth nonlinearities in the throttle valve system, the model predictive controller is designed by approximating the nonlinearity of the throttle valve system.

In summary, the above literatures focuses on how to improve the dynamic performance of the electronic throttle control system. However, the above literature does not take the throttle angle constraint control problem into consideration. In fact, it is important to consider constraints. New design constraint sets are proposed in [12] to control the effective red and effective green durations, such that traffic enters the road lanes without overflow. The opening angles of the throttle plate is restricted by a certain range, such as collision limit $[5,13]$ and return spring, which will be discussed in the paper. Moreover, too much opening angle of the throttle plate will cause the car to stop when the manual car is in low gear, thus causing the accident. Therefore, in order to improve the robustness of the electronic throttle system, it is necessary to constrain the throttle angle within an appropriate range.

At present, constrained control strategy has been studied by some scholars [14-21]. A path tracking control strategy based on linear model predictive control with softening constraints is proposed in [14]. In the optimization process, a relaxation factor is added, and the quadratic programming method is used to calculate the control increment of each cycle. In [15], the multi constraint control problem of hypersonic vehicle is solved, and the robustness of the control system is enhanced by using sliding manifold and disturbance observer. In [16,17], aiming at input and state constraints, a method based on set invariance is proposed. However, the constraint is satisfied only if the initial state is constrained in an invariant set. In [18,19], a governor method is designed to satisfy the constraints of uncertain nonlinear systems, in which the input of the closed-loop system is the reference signal, and the modification of the reference signal does not violate the state and control constraints. Model predictive control can solve the problem of constraint control in a reasonable time and has been widely investigated [20]. In [21], it is demonstrated how the constraints that protect the operating envelope of the engine can be included through the augmentation of a barrier function.

Different from the aforementioned methods, as the variable approaches the constraint boundary, the barrier Lyapunov function (BLF) grows to infinity. Therefore, the BLF is widely used to study the state and output constraints in basic control theory [22-27], and good tracking performance is achieved without violating the constraints. In [22], under the condition that the boundary of BLF and the semi-global boundedness of all closed-loop signals are guaranteed, the tracking error can converge to zero. In [23], without violating the constraints, by ensuring the boundary of the BLF, not only the system asymptotic tracking is realized, but all closed-loop signals are bounded. The output tracking control problem of the lower triangular constrained nonlinear switching system is investigated. In this problem, when all states are constrained, the BLF is used to prevent the state from violating the constraint [24]. In [25], an asymmetric time-varying BLF is adopted 
to ensure that the system satisfies the constraints for strictly feedback nonlinear systems with time-varying output constraints, and a controller is designed. This design method has been successfully applied to many practical control problems [26,27]. An asymmetric BLF controller and an asymmetric time varying BLF controller are designed respectively for the problem of slip rate constraint in the ABS system in [28,29]. A nonlinear position controller is designed in [30] by a backstepping procedure using the barrier Lyapunov function to satisfy the constraints of position error and yaw. In [31], in order to deal with robust stabilization and output constraints of the system, a novel tan-type BLF and a power integrator technique have been proposed. In order to deal with system constraints, BLF is used in the backstepping process, and the control input is regarded as the extended state variable.

Based on the above methods, BLF is introduced into ETCS to solve the problem of desired throttle angle tracking with asymmetric throttle angle constraint. The main contributions of this paper can be summarized as follows:

(1) In the ETCS, we consider a constrained control problem with asymmetric throttle angle constraint to ensure that it does not violate the throttle angle constraint;

(2) The principle of throttle opening limitation is analyzed and a nonlinear ETC mathematic model with uncertainties is established. In the process of constraint controller design, BLF is introduced to deal with symmetric output constraints;

(3) The simulation results show that our BLF control algorithm is more effective than the quadratic Lyapunov function (QLF) control algorithm.

Notation 1. The notations used in this paper are standard. Superscript ' $T$ ' denotes matrix transposition; and $\|X\|$ stands for the Euclidean norm for vectors and the spectral norm for matrices.

\section{Materials and Methods}

\subsection{Electronic Throttle Mathematics Model}

Electronic throttle is mainly composed of electronic control unit, drive motor, reduction gear, throttle body, return spring, and valve plate [6]. Its structure is shown in Figure 1.

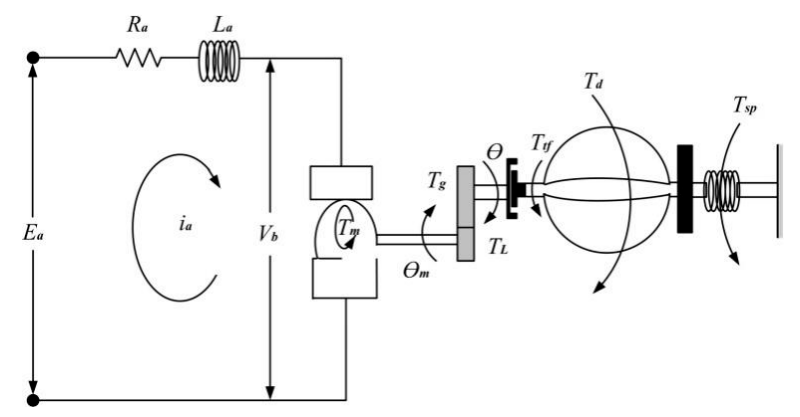

Figure 1. The structure of electronic throttle.

The parameters in the structure are listed in Table 1.

Table 1. Parameters of the Throttle Model.

\begin{tabular}{cc}
\hline Description & Symbol \\
\hline Coefficient of sliding friction & $k_{f}$ \\
Static friction coefficient & $k_{t f}$ \\
Spring modulus & $k_{s p}$ \\
The default opening of the throttle & $\theta_{0}$ \\
Air flow load torque & $T_{d}$ \\
\hline
\end{tabular}


Table 1. Cont.

\begin{tabular}{cc}
\hline Description & Symbol \\
\hline Transmission ratio & $n$ \\
Motor counter emf constant & $k_{b}$ \\
Electrical resistance & $R_{a}$ \\
Motor torque constant & $k_{t}$ \\
Motor input voltage & $E_{a}$ \\
Motor moment of inertia & $J_{m}$ \\
Throttle plate moment of inertia throttle & $J_{n}$ \\
Equivalent moment of inertia & $J$ \\
Throttle angle rotation & $\theta$ \\
Motor shaft damping coefficient & $k_{m}$ \\
Reset spring preload torque coefficient & $k_{\text {pre }}$ \\
\hline
\end{tabular}

In the Reference [6], in order to improve the tracking performance of automotive electronic throttle, a control-oriented nonlinear electronic throttle model is proposed. The system model can be expressed as follows:

$$
\ddot{\theta}=\frac{1}{J}\left[-k_{s p}\left(\theta-\theta_{0}\right)-k_{t f} \operatorname{sgn}(\dot{\theta})+n^{2} k_{t} \frac{E_{a}}{R_{a}}-\left(n^{2} k_{m}+k_{f}+\frac{n^{2} k_{b} k_{t}}{R_{a}}\right) \dot{\theta}\right]-\frac{T_{d}}{J}
$$

where $J=n^{2} J_{m}+J_{g}$.

However, in this paper, the throttle return spring torque $T_{s p}$ neglected the nonlinearity of the tightening torque coefficient of the spring and was given by

$$
T_{s p}=k_{s p}\left(\theta-\theta_{0}\right)
$$

However, the movement of the valve plate is limited between the maximum and minimum values. The limited travel is achieved by the return spring, and ideally there is only limited gain. The characteristic of the modeled spring can be described as

$$
T_{s p}=\left\{\begin{array}{c}
T_{L H}+k_{\text {pre }}\left(\theta-\theta_{0}\right), \text { if } \theta_{0} \leq \theta \leq \theta_{\max } \\
-T_{L H}+k_{p r e}\left(\theta-\theta_{0}\right), \text { if } \theta_{\min } \leq \theta \leq \theta_{0}
\end{array}\right.
$$

where $T_{L H}$ is the spring offset and $\theta_{\min }$ and $\theta_{\max }$ are the lower and upper bounds of the throttle opening angle, respectively.

So, we can have

$$
T_{s p}=k_{s p}\left(\theta-\theta_{0}\right)+k_{p r e} \operatorname{sig}\left(\theta-\theta_{0}\right)
$$

Therefore, the system model can be rewritten as

$$
\ddot{\theta}=\frac{1}{J}\left[-k_{s p}\left(\theta-\theta_{0}\right)-k_{p r e} \operatorname{sig}\left(\theta-\theta_{0}\right)-k_{t f} \operatorname{sgn}(\dot{\theta})+n^{2} k_{t} \frac{E_{a}}{R_{a}}-\left(n^{2} k_{m}+k_{f}+\frac{n^{2} k_{b} k_{t}}{R_{a}}\right) \dot{\theta}\right]-\frac{T_{d}}{J}
$$

Defining state variables as $x_{1}=\theta, x_{2}=\dot{\theta}, u=E_{a}$ and output variable $y=\theta$, the system model can be rewritten as

$$
\left\{\begin{array}{c}
\dot{x}_{1}=x_{2} \\
\dot{x}_{2}=f(x, t)+g(x, t) \cdot u+d(t) \\
y=x_{1}
\end{array}\right.
$$

where

$$
f(x, t)=-\frac{k_{s p}}{J} x_{1}-\frac{n^{2} k_{m}+k_{f}+\frac{n^{2} k_{b} k_{t}}{R_{a}}}{J} x_{2}+\frac{k_{s p}}{J} \theta_{0}-\frac{k_{t f} \operatorname{sgn}\left(x_{2}\right)+T_{d}}{J}+\frac{k_{p r e s g n}\left(\theta_{0}-x_{1}\right)}{J}, g(x, t)=
$$
$\frac{n^{2} k_{t}}{J R_{a}}, d(t)$ represents the sum of modeling error and unknown disturbance uncertainty. The $|d(t)|$ is bounded. 
What we're trying to do is to design a constraint backstepping controller for a nonlinear dynamic system (6), which can make the output $y$ and can track a desired trajectory $y_{d}(t)$ while ensuring the output constraint is not violated. Therefore, a controller based on BLF is designed to suppress the disturbances and handle the symmetric output constraints to improve the ETCS robustness.

\subsection{Problem Statement}

For an electronic throttle control system, the performance requirements to be considered in controller design include the following three aspects.

Firstly, one of the main tasks is to limit the throttle position, getting achievement of transient zero overshoot and avoiding the collision of limited position [5,12]. For the sake of controller performance analysis, we imply the objective is to specify another compact as large as desired for any initial compact set to which $y(0)$ belongs. In other words, the output constraints are not violated.

Secondly, the goal of the nonlinear dynamical system controller is to control the output $y$ can track desired trajectory $y_{d}(t)$.

Lastly, the strategy ensures that all the closed-loop signals are bounded.

\subsection{BLF Preliminaries}

To better establish constraint compensation and performance boundaries, we introduce the following definitions, assumptions, and lemma.

Definition 1 ([23]). A barrier Lyapunov function is a scalar function $V(x)$, defined with respect to the system $\dot{x}=f(x, t)$ on an open region $D$ containing the origin, that is continuous, positive definite, has continuous first-order partial derivatives at every point of $D$, has the property $V(x) \rightarrow \infty$ as $x$ approaches the boundary of $D$, and satisfies $V(x) \leq b, \forall t \geq 0$, along the solution of $\dot{x}=f(x, t)$ for $x(0) \in D$ and some positive constant $b$.

A barrier Lyapunov function should be symmetric or asymmetric according to the boundary character, which is shown in Figure 2.

Assumption 1 ([16]). There exist positive constants $Y_{1}, Y_{2}$ and functions $\underline{Y}_{0}(t), \bar{Y}_{0}(t)$ satisfying $\underline{Y}_{0}(t)>\underline{k}_{c 1}(t), \bar{Y}_{0}(t)<\bar{k}_{c 1}(t)$, such that the desired trajectory $y_{d}(t)$ and its time derivatives satisfy $\underline{Y}_{0}(t) \leq y_{d}(t) \leq \bar{Y}_{0}(t)$ and $\left|\dot{y}_{d}(t)\right| \leq Y_{1},\left|\ddot{y}_{d}(t)\right| \leq Y_{2}, \forall t \geq 0$, implying that they are continuous and available in a compact set $\Omega y_{d}:=\left\{\left[y_{d}, \dot{y}_{d}, \ddot{y}_{d}\right]^{T}: y_{d}^{2}+\dot{y}_{d}^{2}+\ddot{y}_{d}^{2} \leq \delta_{y d}\right\} \subset \mathbb{R}^{3}$.

Lemma 1 ([32]). For any positive functions $k_{a 1}(t), k_{b 1}(t)$, let $S_{1}=\left\{S_{1} \in \mathbb{R} \mid-k_{a 1}(t)<S_{1}<k_{b 1}(t)\right\}$ $\subset \mathbb{R}$, and $N:=\mathbb{R}^{l} \times S_{1} \subset \mathbb{R}^{l+1}$ be open sets. Consider the system

$$
\dot{\eta}=h(t, \eta)
$$

where $\eta:=\left[\begin{array}{ll}\omega & S_{1}\end{array}\right]^{T} \in N$, and $h:=\mathbb{R}_{+} \times N \rightarrow \mathbb{R}^{l+1}$ is piecewise continuous with respect to $t$ and locally Lipschitz with respect to $\eta$, uniformly with respect to $t$, on $\mathbb{R}_{+} \times N$. Suppose that there exist functions $U: \mathbb{R}^{l} \rightarrow \mathbb{R}_{+}$and $V_{1}: S_{1} \rightarrow \mathbb{R}_{+}$continuously differentiable and positive definite in their respective domains, such that

$$
V_{1}(z) \rightarrow \infty \text { as } z \rightarrow-k_{a 1}(t) \text { or } z \rightarrow k_{b 1}(t) \gamma_{1}(\|\omega\|) \leq \mathrm{U}(\omega) \leq \gamma_{2}(\|\omega\|)
$$

where $\gamma_{1}$ and $\gamma_{2}$ are class $K_{\infty}$ functions. Let $V(\eta):=V_{1}\left(S_{1}\right)+U(\omega)$ and $S_{1}(0) \in S_{1}$. If the inequality holds

$$
\dot{V}=\frac{\partial V}{\partial \eta} h \leq-c V+v
$$

in the set $\eta \in N$ and $c, v$ are positive constants, then $S_{1}(t)$ remains in the open set $S_{1}, \forall t \in[0, \infty]$. 

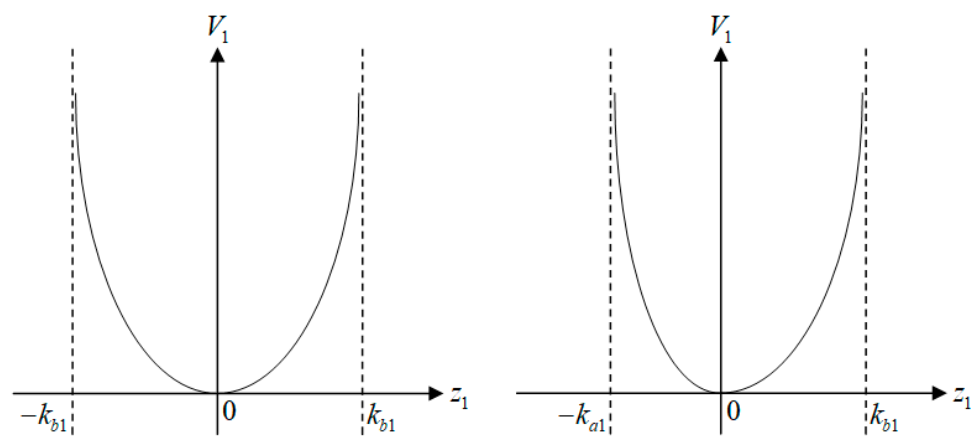

Figure 2. Symmetric (left) and asymmetric (right) barrier Lyapunov function.

\section{Controller Design}

In the process of controller design in this section, fixed throttle angle constraints are directly considered to ensure that the constraint boundary is not exceeded. We employ the ABLF to design controller.

Defining the fixed constraints $k_{a 1}, k_{b 1}$ as

$$
\left\{\begin{array}{l}
k_{a 1}=Y_{l}-\underline{k}_{c} \\
k_{b 1}=\bar{k}_{c}-Y_{h}
\end{array}\right.
$$

where $Y_{l}$ and $Y_{h}$ are the minimum and maximum value of the desired throttle angle $\theta^{*}$, respectively. $\underline{k}_{c}$ and $\bar{k}_{c}$ are the lower and upper bounds of opening angles of the throttle plate, respectively. The algorithm can be written as follows:

Step1: Denote $z_{1}=x_{1}-\theta^{*}$ as the tracking error, and $z_{2}=x_{2}-\alpha_{1}$ as a virtual error, where $\alpha_{1}$ is a virtual control function.

By selecting the asymmetric barrier Lyapunov function,

$$
V_{1}\left(z_{1}\right)=\frac{1}{2}\left(1-q\left(z_{1}\right)\right) \log \frac{k_{a 1}^{2}}{k_{a 1}^{2}-z_{1}^{2}}+\frac{1}{2} q\left(z_{1}\right) \log \frac{k_{b 1}^{2}}{k_{b 1}^{2}-z_{1}^{2}}
$$

where

$$
q\left(z_{1}\right)=\left\{\begin{array}{c}
1,0<z_{1}<k_{b 1} \\
0,-<k_{a 1} z_{1} \leq 0
\end{array}\right.
$$

$k_{a 1}$ and $k_{b 1}$ are the asymmetric constraints on $z_{1}$, and each of these can be set independently, depending on the upper and lower bounds of $\theta^{*}$. To simplify the problem, we will denote $q\left(z_{1}\right)$ by $q$.

By taking the derivative of Equation (8), we get the following expression:

$$
\dot{V}_{1}\left(z_{1}\right)=(1-q) \frac{z_{1} \dot{z}_{1}}{k_{a 1}^{2}-z_{1}^{2}}+q \frac{z_{1} \dot{z}_{1}}{k_{b 1}^{2}-z_{1}^{2}}=\left(\frac{1-q}{k_{a 1}^{2}-z_{1}^{2}}+\frac{q}{k_{b 1}^{2}-z_{1}^{2}}\right) z_{1} \dot{z}_{1}
$$

Because $z_{1}=x_{1}-\theta^{*}$, so we can have $\dot{z}_{1}=\dot{x}_{1}-\dot{\theta}^{*}=x_{2}-\dot{\theta}^{*}=z_{2}+\alpha_{1}-\dot{\theta}^{*}$. So, we can have

$$
\dot{V}_{1}\left(z_{1}\right)=\left(\frac{1-q}{k_{a 1}^{2}-z_{1}^{2}}+\frac{q}{k_{b 1}^{2}-z_{1}^{2}}\right) z_{1}\left(z_{2}+\alpha_{1}-\dot{\theta}^{*}\right)
$$

Design the virtual controller $\alpha_{1}$ as

$$
\alpha_{1}=-k_{1}\left[(1-q)\left(k_{a 1}^{2}-z_{1}^{2}\right)+q\left(k_{b 1}^{2}-z_{1}^{2}\right)\right] z_{1}+\dot{\theta}^{*}
$$

where $k_{1}$ is a positive constant. 
Equation (12) is substituted into (11) to obtain

$$
\dot{V}_{1}\left(z_{1}\right)=-k_{1} z_{1}^{2}+\left(\frac{1-q}{k_{a 1}^{2}-z_{1}^{2}}+\frac{q}{k_{b 1}^{2}-z_{1}^{2}}\right) z_{1} z_{2}
$$

when $z_{2} \rightarrow 0$, there is $\dot{V}_{1}\left(z_{1}\right)=-k_{1} z_{1}^{2}$ and $\dot{V}_{1}\left(z_{1}\right) \rightarrow 0$ when $t \rightarrow \infty$. The term $\left(\frac{1-q}{k_{a 1}^{2}-z_{1}^{2}}+\frac{q}{k_{b 1}^{2}-z_{1}^{2}}\right) z_{1} z_{2}$ can be canceled in a later step.

Step2: Define the Lyapunov function candidate as

$$
V=V_{1}\left(z_{1}\right)+\frac{1}{2} z_{2}^{2}+\frac{1}{2 \gamma} \widetilde{\eta}^{2}
$$

where $\widetilde{\eta}=\hat{\eta}-\eta$ is the estimation of uncertainty upper bound $\eta$ and $\eta \geq|d(t)|$, and $\gamma$ is a positive constant.

Differentiating Equation (14), we can obtain

$$
\begin{aligned}
\dot{V} & =\dot{V}_{1}\left(z_{1}\right)+z_{2} \dot{z}_{2}+\frac{1}{\gamma} \widetilde{\eta} \dot{\tilde{\eta}} \\
& =-k_{1} z_{1}^{2}+\left(\frac{1-q}{k_{a 1}^{2}-z_{1}^{2}}+\frac{q}{k_{b 1}^{2}-z_{1}^{2}}\right) z_{1} z_{2}+z_{2}\left[f(x, t)+g(x, t) \cdot u+d(t)-\dot{\alpha}_{1}\right]+\frac{1}{\gamma} \widetilde{\eta} \dot{\eta}
\end{aligned}
$$

The adaptive control law can be written as

$$
u=\frac{1}{g(x, t)}\left[-\left(\frac{1-q}{k_{a 1}^{2}-z_{1}^{2}}+\frac{q}{k_{b 1}^{2}-z_{1}^{2}}\right) z_{1}-k_{2} z_{2}-f(x, t)+\dot{\alpha}_{1}-\hat{\eta} \operatorname{sign}\left(z_{2}\right)\right]
$$

where $k_{2}$ is a positive constant.

Equation (16) is substituted into (15) to obtain

$$
\begin{aligned}
\dot{V} & =-k_{1} z_{1}^{2}-k_{2} z_{2}^{2}+z_{2}\left(d(t)-\hat{\eta} \operatorname{sign}\left(z_{2}\right)\right)+\frac{1}{\gamma} \widetilde{\eta} \dot{\tilde{\eta}} \\
& =-k_{1} z_{1}^{2}-k_{2} z_{2}^{2}+z_{2}\left(d(t)-\eta \operatorname{sign}\left(z_{2}\right)-\widetilde{\eta} \operatorname{sign}\left(z_{2}\right)\right)+\frac{1}{\gamma} \tilde{\eta} \dot{\hat{\eta}} \\
& =-k_{1} z_{1}^{2}-k_{2} z_{2}^{2}-z_{2}\left(\eta \operatorname{sign}\left(z_{2}\right)-d(t)\right)-z_{2} \widetilde{\eta} \operatorname{sign}\left(z_{2}\right)+\frac{1}{\gamma} \tilde{\eta} \dot{\hat{\eta}} \\
& =-k_{1} z_{1}^{2}-k_{2} z_{2}^{2}-z_{2}\left(\eta \operatorname{sign}\left(z_{2}\right)-d(t)\right)-\widetilde{\eta}\left(\left|z_{2}\right|-\frac{1}{\gamma} \dot{\hat{\eta}}\right)
\end{aligned}
$$

The adaptive law can be designed as follows:

$$
\dot{\hat{\eta}}=\gamma\left|z_{2}\right|
$$

By substituting (18) into (17), we can obtain

$$
\begin{aligned}
\dot{V} & =-k_{1} z_{1}^{2}-k_{2} z_{2}^{2}-z_{2}\left(\eta \operatorname{sign}\left(z_{2}\right)-d(t)\right) \\
& =-k_{1} z_{1}^{2}-k_{2} z_{2}^{2}-\left(z_{2} \operatorname{sign}\left(z_{2}\right) \eta-z_{2} d(t)\right) \\
& \leq-k_{1} z_{1}^{2}-k_{2} z_{2}^{2}-\left|z_{2}\right|(\eta-|d(t)|) \\
& \leq 0
\end{aligned}
$$

As can be seen in Equation (19), $\dot{V}$ is negative definite when the control law is (16) and the adaptive law is (18). Thus, the system is asymptotically stable. When $t \rightarrow \infty$, the $z_{1} \rightarrow 0$. Based on Lemma $1, z_{1}$ remains in $\left(-k_{a 1}, k_{b 1}\right), \forall t \in[0, \infty]$. Therefore, the throttle angle constraints will never be violated.

\section{Discussion}

In this part, simulation is carried out for three different conditions to verify the effectiveness of the proposed control strategy. Throttle Model Parameters are given in Table 2. 
Table 2. Throttle Model Parameters.

\begin{tabular}{ccc}
\hline Description & Symbol & Value \\
\hline Coefficient of sliding friction & $k_{f}$ & $4.0 \times 10^{-4} \frac{\mathrm{N} \times \mathrm{m}}{\mathrm{rad} / \mathrm{s}}$ \\
Static friction coefficient & $k_{t f}$ & $0.0048 \mathrm{Nm}$ \\
Spring modulus & $k_{s p}$ & $0.0247 \frac{\mathrm{N} \times \mathrm{m}}{\mathrm{rad}}$ \\
The default opening of the throttle & $\theta_{0}$ & $0.0349 \mathrm{rad}$ \\
Air flow load torque & $T_{d}$ & $0.02 \mathrm{~N} \times \mathrm{m}$ \\
Transmission ratio & $n$ & 16.95 \\
Motor counter emf constant & $k_{b}$ & $0.016 \frac{\mathrm{V}}{\mathrm{rad} / \mathrm{s}}$ \\
Electrical resistance & $R_{a}$ & $2.8 \Omega \mathrm{N}$ \\
Motor torque constant & $k_{t}$ & $0.016 \mathrm{~N} \times \mathrm{A}^{-1}$ \\
Equivalent moment of inertia & $J$ & $1.15 \times 10^{-3} \mathrm{~kg} \times \mathrm{m}^{2}$ \\
Motor shaft damping coefficient & $k_{m}$ & $1.6 \times 10^{-6} \frac{\mathrm{N} \times \mathrm{m}}{\mathrm{rad} / \mathrm{s}}$ \\
Reset spring preload torque & $k_{p r e}$ & $0.107 \mathrm{~N} \times \mathrm{m}$ \\
coefficient & &
\end{tabular}

Case 1: Under the simulated fixed gear condition, the expected value of throttle opening and the boundary of throttle opening are fixed.

Case 2: Under the simulated shifting condition, the expected value of throttle opening and the boundary of throttle opening are switched. However, under each fixed gear condition, the expected value of throttle opening and the boundary of throttle opening are fixed.

Case 3: Under the simulated fixed gear condition, the expected value of throttle opening is a positive curve and the boundary of throttle opening is fixed.

\subsection{Case 1}

In this sub-section, expected value of throttle opening is 0.4 rad step signal, the upper boundary of throttle opening is 0.5 , and the lower boundary of throttle opening is 0 . System uncertainty satisfies $d(t)=0.1 \cos (t)$. The parameters are $k_{1}=190, k_{2}=150$, $\gamma=1, Y_{l}=Y_{h}=0.4, \underline{k}_{c}=0$, and $\bar{k}_{c}=0.5$. States variable initial value: $\mathbf{x}(0)=\left[\begin{array}{ll}0.1 & 0\end{array}\right]^{T}$. So, we can have $k_{a}=Y_{l}-\underline{k}_{c}=0.4$ and $k_{b}=\bar{k}_{c}-Y_{h}=0.1$. The simulation results can be seen in Figure 3.

Figure 3a shows the tracking response curve of the system when the expected throttle opening is $0.4 \mathrm{rad}$, and Figure $3 \mathrm{~b}$ shows the tracking error in this process. From Figure $3 \mathrm{a}, \mathrm{b}$, the ABLF controller can obtain good throttle opening tracking performance, the actual throttle opening is strictly kept within the throttle angle constraint boundary $(0,0.5)$, and the tracking error is within the constraint $(-0.4,0.1)$. This implies the ETCS will impossibly collide with the limit position and control transient overshoot effectively, even zero overshoot. The stability time of the step response is less than 150 milliseconds, and the stability error approaches zero gradually. However, QLF controller not only exceeds the constraint boundary of throttle opening angle, but also exceeds the constraint boundary of error, which means that the excessive transient overshoot of the desired throttle angle tracking can result in colliding limit position. At same time, the throttle valve fluctuates sharply, seriously impacting on the lifespan of the actuator. In addition, too much opening angle of the throttle plate will cause the car to stop when the manual car is in low gear, which will lead to accidents. 


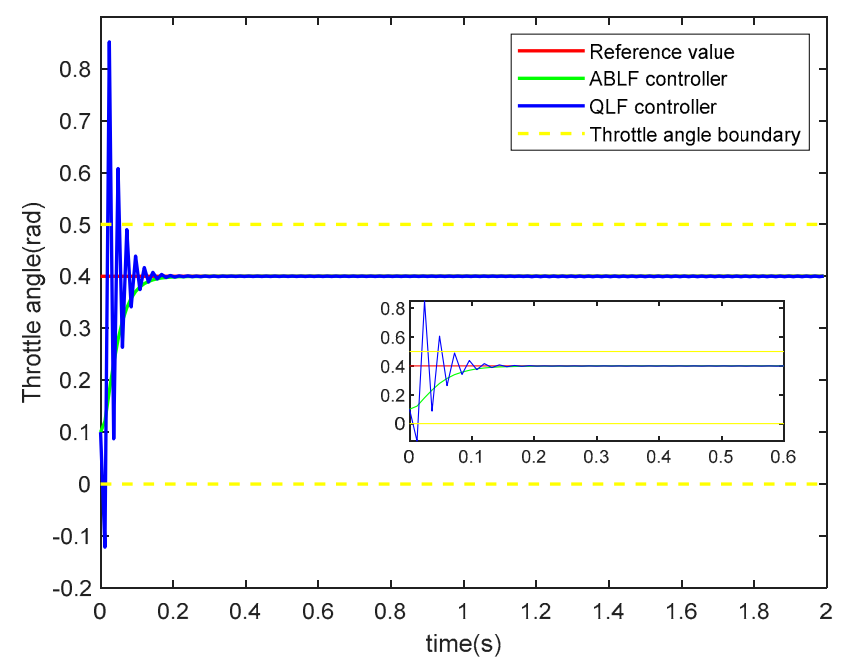

(a) Throttle angle.

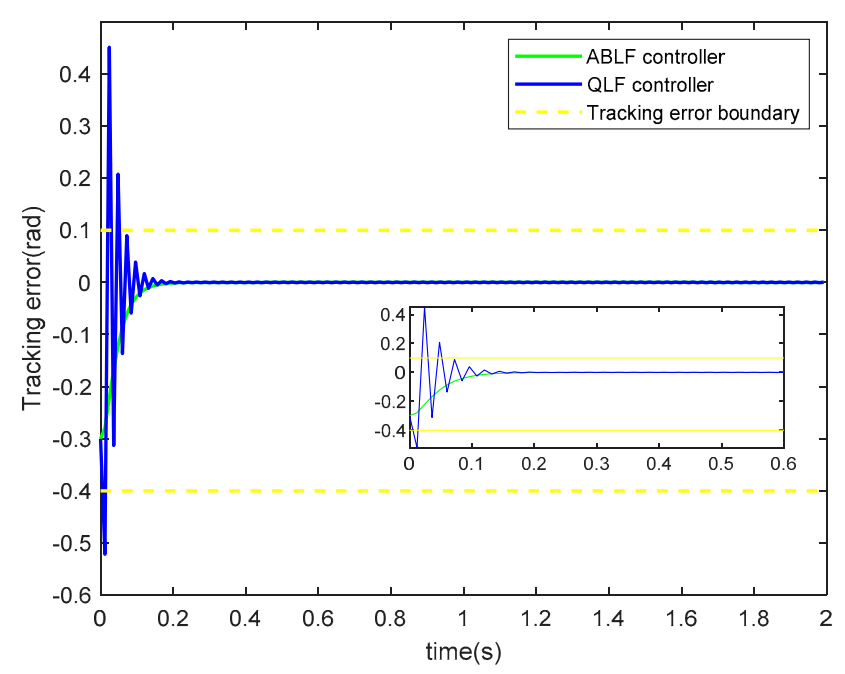

(b) Tracking error.

Figure 3. Simulation results under step signal: (a) Throttle angle, (b) Tracking error.

\subsection{Case 2}

In this sub-section, expected value of throttle opening is jump signal.

In $0-0.6 \mathrm{~s}$, expected value of throttle opening is $0.8 \mathrm{rad}$, the upper boundary of throttle opening is 0.9 , the lower boundary of throttle opening is 0 . System uncertainty satisfies $d(t)=0.1 \cos (t)$. The controller parameters are $k_{1}=190, k_{2}=150, \gamma=1, Y_{l}=Y_{h}=0.8$, $\underline{k}_{c}=0$, and $\bar{k}_{c}=0.9$. States variable initial value: $\mathbf{x}(0)=\left[\begin{array}{ll}0.1 & 0\end{array}\right]^{T}$. So, we can have $k_{a}=Y_{l}-\underline{k}_{c}=0.8$ and $k_{b}=\bar{k}_{c}-Y_{h}=0.1$.

In $0.6-0.7 \mathrm{~s}$, the system is in the process of shifting. The expected value of throttle opening and the boundary of throttle opening are switched.

In $0.7-5 \mathrm{~s}$, expected value of throttle opening is $0.4 \mathrm{rad}$, the upper boundary of throttle opening is 0.5 , and the lower boundary of throttle opening is 0 . System uncertainty satisfies $d(t)=0.1 \cos (t)$. The controller parameters are chosen as follows: $k_{1}=190, k_{2}=150$, $\gamma=1, Y_{l}=Y_{h}=0.4, \underline{k}_{c}=0$, and $\bar{k}_{c}=0.5$. So, we can have $k_{a}=Y_{l}-\underline{k}_{c}=0.4$ and $k_{b}=\bar{k}_{c}-Y_{h}=0.1$.

Note: Since the derivative of the step function tends to infinity as it passes from one gear to another, Assumption 1 is not satisfied. In this paper, the transition part of the step function is replaced by a linear function corresponding to a line segment near the 
vertical line, as shown in Figure 4a. This satisfies both the actual moving process and the Assumption 1.

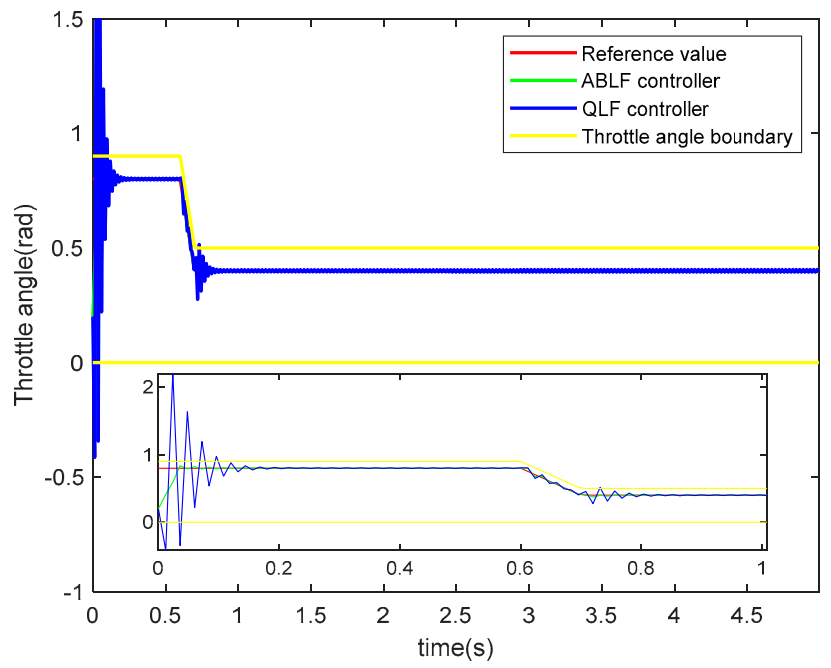

(a) Throttle angle.

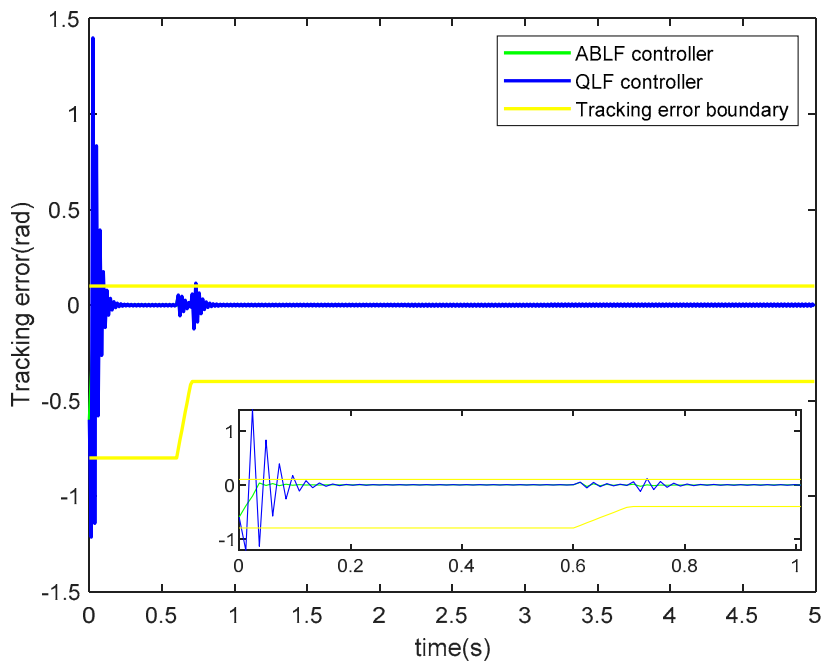

(b) Tracking error.

Figure 4. Simulation results under jump signal: (a) Throttle angle, (b) Tracking error.

The simulation results can be seen in Figure 4 .

Note from Figure $4 \mathrm{a}, \mathrm{b}$ that actual throttle opening angle is always within the constraint range $(0,0.9)$ and the tracking error is in the constrained range $(-0.8,0.1)$ before shift. After shift, the actual throttle opening stays strictly within the throttle angle constraint range $(0,0.5)$ and the tracking error is in the constrained range $(-0.4,0.1)$. Therefore, the ABLF control method achieves good tracking performance. The actual throttle angle is always within the constraint range, and the error is always within the constraint range in the whole braking process. Furthermore, the tracking curve is smooth during the shifting condition.

However, the QLF controller not only exceeds the constraint boundary of throttle opening angle, but also exceeds the constraint boundary of error, which means that the excessive transient overshoot of the desired throttle angle tracking can result in colliding limit position. At same time, the throttle valve exist fluctuations during the shifting, impacting on the lifespan of the actuator seriously. 


\subsection{Case 3}

In this sub-section, expected value of throttle opening is $(0.4+0.1 \cdot \sin (2 t)) \mathrm{rad}$, the upper boundary of throttle opening is 0.65 , and the lower boundary of throttle opening is 0 . System uncertainty satisfies $d(t)=0.1 \cos (t)$. The controller parameters are $k_{1}=190$, $k_{2}=150, \gamma=1, Y_{l}=0.3, Y_{h}=0.5, \underline{k}_{c}=0$, and $\bar{k}_{c}=0.65$. States variable initial value: $\mathbf{x}(0)=\left[\begin{array}{ll}0.12 & 0\end{array}\right]^{T}$. So, we can have $k_{a}=Y_{l}-\underline{k}_{c}=0.3$ and $k_{b}=\bar{k}_{c}-Y_{h}=0.15$. The simulation results are shown in Figure 5.

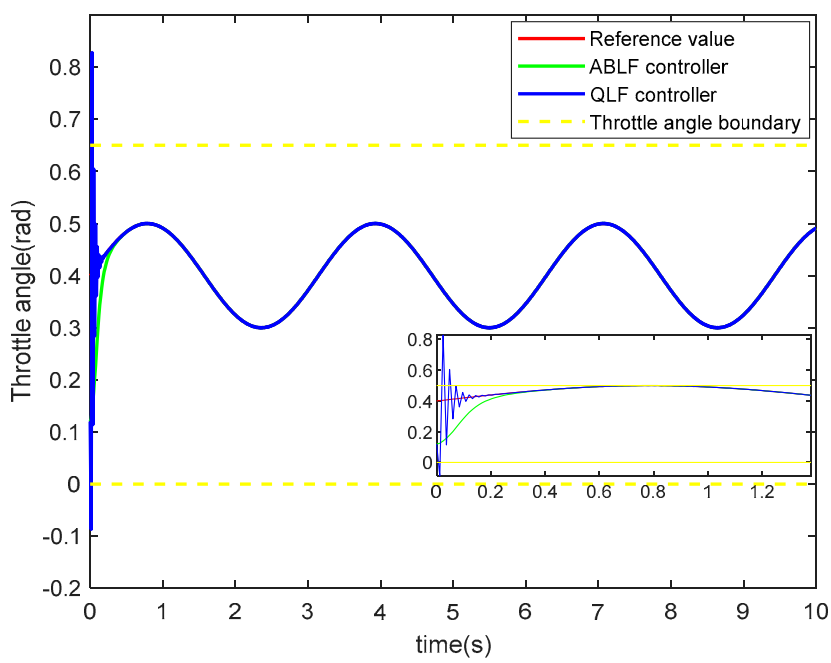

(a) Throttle angle.

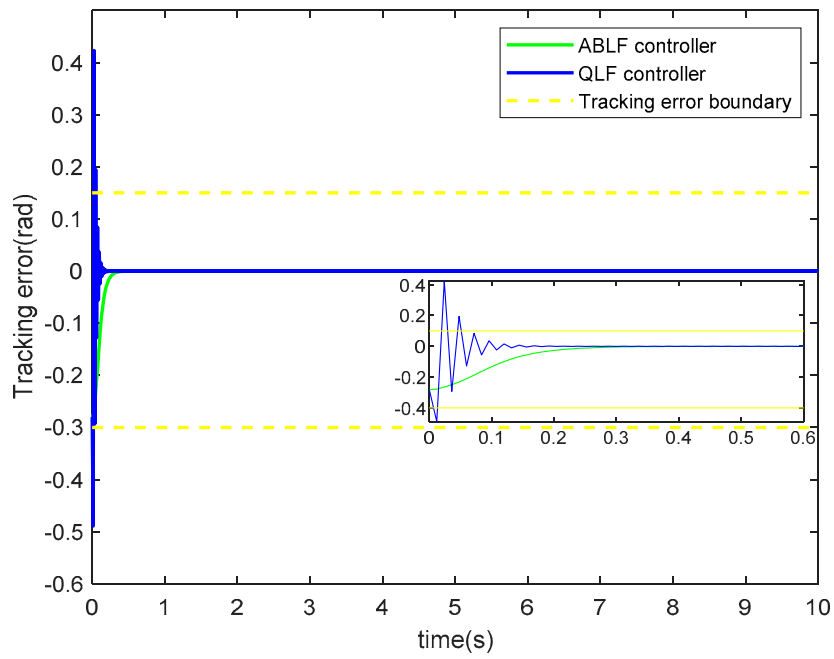

(b) Tracking error.

Figure 5. Simulation results under sinusoidal signal: (a) Throttle angle, (b) Tracking error.

It can be seen from Figure $5 \mathrm{a}, \mathrm{b}$ that the ABLF controller can receive good tracking performance of expected throttle opening, actual throttle opening angle is always within the constraint range $(0,0.65)$, and the tracking error is strictly within the error constraint range $(-0.3,0.15)$. This implies the ETCS will impossibly collide with the limit position and control transient overshoot effectively, even zero overshoot. The stability time of the step response is less than 250 milliseconds, and the stability error approaches zero gradually. However, the QLF controller not only exceeds the constraint boundary of throttle opening angle, but also exceeds the constraint boundary of error, which means that the 
excessive transient overshoot of the desired throttle angle tracking can result in colliding limit position.

As can be seen in Table 3, the values of the performance criteria-over-adjustment, adjustment time, adjustment error —of the adjustment system in the studied cases are clear. The BLF control strategies achieve the requirement: it has a shorter adjustment time, no over-adjustment, no violation of boundary, and smaller maximum absolute tracking error. However, with the QLF controller, the boundary of the throttle opening angle and tracking error are violated with serious over-adjustment.

Table 3. The Performance Comparison.

\begin{tabular}{ccccc}
\hline Case & Strategy & Adjustment Time & Over-Adjustment & Adjustment Error \\
\hline \multirow{2}{*}{1} & QLF & 0.17 & $115 \%$ & 0.44 \\
& BLF & 0.16 & 0 & 0.3 \\
2 & QLF & 0.18 & $87.5 \%$ & 1.4 \\
& BLF & 0.02 & $5 \%$ & 0.6 \\
3 & QLF & 0.18 & $97.6 \%$ & 0.43 \\
& BLF & 0.3 & 0 & 0.3 \\
\hline
\end{tabular}

\section{Conclusions}

In this paper, a second order mathematical model of ETCS is proposed, and a constraint controller based on ABLF is designed to constrain the throttle angle within an appropriate range to avoid collision with the limit position. The proposed controller is compared with the controller based on QLF via simulation of the analysis of different signal wave tracking response performance, such as step signal, jump signal, and sinusoidal signal. The simulation results show that the controller we designed can realize zero error tracking of throttle angle, and the constraint boundaries of throttle angle can not be violated under different response signals. In contrast, QLF-based controllers can result in constraint boundary violations. This means that the excessive transient overshoot of the desired throttle angletracking can result in colliding limit position. In addition, compared with the control effect of QLF controller, the controller we designed will not make the throttle angle oscillate. Because of the constraint boundary, the controller is more complex and the calculation is more complicated than the traditional PID or other methods. However, when the constraint boundary is taken into account, the motion angle range of the electronic throttle is limited to a reasonable range. The electronic throttle does not collide with the limit position due to frequent over-adjustment, which will increase the life of the actuator. Moreover, such precise control will make air intake more accurate, enable the engine airfuel ratio to be accurately controlled, save fuel, and protect the environment. It is therefore worth further research.

Author Contributions: Conceptualization, D.W. and S.L.; methodology, Y.H.; software, Y.H.; validation, D.W., S.L., and Y.H.; formal analysis, S.L.; investigation, J.S.; resources, S.L.; data curation, J.S.; writing—original draft preparation, D.W.; writing—review and editing, J.S.; visualization, Y.H.; supervision, J.S.; project administration, D.W.; funding acquisition, Y.H. All authors have read and agreed to the published version of the manuscript.

Funding: This work is supported by Jiangsu province "Six Talent Peaks" project (2015-XNYQC-004), Jiangsu province road transport application Key Laboratory Fund (BM20082061506), and a research start fund of Jiangsu University (15JDG125).

Institutional Review Board Statement: Not applicable.

Informed Consent Statement: Not applicable.

Data Availability Statement: Not applicable.

Conflicts of Interest: The authors declare no conflict of interest. 


\section{References}

1. Wang, H.; Yuan, X.; Wang, Y.; Yang, Y. Harmony search algorithm-based fuzzy-PID controller for electronic throttle valve. Neural Comput. Appl. 2013, 22, 329-336. [CrossRef]

2. Li, Y.; Yang, B.; Zheng, T.; Li, Y. Extended State Observer Based Adaptive Back-Stepping Sliding Mode Control of Electronic Throttle in Transportation Cyber-Physical Systems. Math. Probl. Eng. 2015. [CrossRef]

3. Ahmed, S.; Badri, A.S.; Mshari, M.H. Integral Sliding Mode Control Design for Electronic Throttle Valve. Eng. J. 2015, 11, 72-84.

4. Bai, R. Adaptive Sliding-Mode Control of an Automotive Electronic Throttle in the Presence of Input Saturation Constraint. IEEE/CAA J. Autom. Sin. 2018, 5, 116-122. [CrossRef]

5. Zheng, P.T.; Sun, J.M. Convergence Back-Stepping Controller Design of Electronic Throttle in Limited Time. Automob. Technol. 2018, 2018, 27-31. (In Chinese)

6. Chen, H.; Hu, Y.F.; Guo, H.Z.; Song, T.-H. Control of electronic throttle based on backstepping approach. Control Theory Appl. 2011, 28, 91-496, 503.

7. Nobuo, K.; Hiroyuki, Y. Adaptive Back-Stepping Control of Automotive Electronic Control Throttle. J. Softw. Eng. Appl. 2017, 10, 41-55.

8. Nobuo, K. Back-Stepping Control of Automotive Electronic Throttle. In Proceedings of the SICE 2015-54th Annual Conference of the Society of Instrument and Control Engineers, Hangzhou, China, 28-30 July 2015.

9. Wang, C.H.; Huang, D.Y. A New Intelligent Fuzzy Controller for Nonlinear Hysteretic Electronic Throttle in Modern Intelligent Automobiles. IEEE Trans. Ind. Electron. 2013, 60, 2332-2345. [CrossRef]

10. İkbal, E.; Şahin, Y. Neural network-based fuzzy inference system for speed control of heavy duty vehicles with electronic throttle control system. Neural Comput. Appl. 2016, 28, 907-916.

11. Khinchi, A.; Prasad, M.P.R. Control of electronic throttle valve using model predictive control. Int. J. Adv. Technol. Eng. Explor. 2016, 3, 118-124. [CrossRef]

12. Wong, C.-K.; Lee, Y.-Y. Lane-Based Traffic Signal Simulation and Optimization for Preventing Overflow. Mathematics 2020, 8, 1368. [CrossRef]

13. Paul, G.G. Embedded Software Control Design for an Electronic Throttle Body. Ph.D. Thesis, University of California, Berkekey, CA, USA, 2002.

14. Ye, H.; Jiang, H.; Ma, S.; Tang, B.; Wahab, L. Linear model predictive control of automatic parking path tracking with soft constraints. Int. J. Adv. Robot. Syst. 2019, 16. [CrossRef]

15. Zhang, J.; Sun, T. Disturbance observer-based sliding manifold predictive control for reentry hypersonic vehicles with multiconstraint. Proc. Inst. Mech. Eng. Part G J. Aerosp. Eng. 2016, 230, 485-495. [CrossRef]

16. Hu, T.; Lin, Z.; Chen, B.M. An analysis and design method for linear systems subject to actuator saturation and disturbance. Automatica 2002, 38, 351-359. [CrossRef]

17. Hu, T.; Lin, Z. Composite Quadratic Lyapunov Functions for Constrained Control Systems. IEEE Trans. Autom. Control 2003, 48, 440-450.

18. Gilbert, E.; Kolmanovsky, I. Nonlinear tracking control in the presence of state and control constraints: A Generalized Reference Governor. Automatica 2002, 38, 2063-2073. [CrossRef]

19. KogisoK; Hirata, K. Reference governor for constrained systems with time-varying references. Robot. Auton. Syst. 2009, 57, 289-295. [CrossRef]

20. Bemporad, A.; Borrelli, F.; Morari, M. Model Predictive Control Based on Linear Programming-The Explicit Solution. IEEE Trans. Autom. Control 2002, 47, 1974-1985. [CrossRef]

21. Kolmanovsky, I.V.; Druzhinina, M.; Sun, J. Speed-gradient approach to torque and air-to-fuel ratio control in DISC engines. Control Syst. Technol. IEEE Trans. 2002, 10, 671-678. [CrossRef]

22. Ren, B.; Sam Ge, S.; Tee, K.P.; Lee, T.H. Adaptive Neural Control for Output Feedback Nonlinear Systems Using a Barrier Lyapunov Function. IEEE Trans. Neural Netw. 2010, 21, 1339-1345. [PubMed]

23. Tee, K.P.; Ge, S.S.; Tay, E.H. Barrier Lyapunov Functions for the control of output-constrained nonlinear systems. Automatica 2009, 45, 918-927. [CrossRef]

24. Niu, B.; Zhao, J. Barrier Lyapunov functions for the output tracking control of constrained nonlinear switched systems. Syst. Control Lett. 2013, 62, 963-971. [CrossRef]

25. Tee, K.P.; Ren, B.; Sam Ge, S. Control of nonlinear system with time-varying output constraints. Automatica 2011, 47, 2511-2516. [CrossRef]

26. Ma, L.; Li, D. Adaptive Neural Networks Control Using Barrier Lyapunov Functions for DC Motor System with Time-Varying State Constraints. Complexity 2018. [CrossRef]

27. Han, S.I.; Cheong, J.Y.; Lee, J.M. Barrier Lyapunov Function-Based Sliding Mode Control for Guaranteed Tracking Performance of Robot Manipulator. Math. Probl. Eng. 2013, 2013, 633-654. [CrossRef]

28. He, Y.G.; Lu, C.D.; Shen, J.; Yuan, C. Design and analysis of output feedback constraint control for antilock braking system with time-varying slip ratio. Math. Probl. Eng. 2019. [CrossRef]

29. He, Y.G.; Lu, C.D.; Shen, J.; Yuan, C. Design and analysis of output feedback constraint control for antilock braking system based on Burckhardt's model. Assem. Autom. 2019, 39, 497-513. [CrossRef] 
30. Kim, W.; Shin, D.; Lee, Y. Nonlinear Position Control Using Only Position Feedback under Position Errors and Yaw Constraints for Air Bearing Planar Motors. Mathematics 2020, 8, 1354. [CrossRef]

31. Fang, L.; Ma, L.; Ding, S.; Zhao, D. Robust finite-time stabilization of a class of high-order stochastic nonlinear systems subject to output constraint and disturbances. Int. J. Robust Nonlinear Control 2019, 29, 5550-5573. [CrossRef]

32. Slotine, J.; Li, W. Applied Nonlinear Control, 1st ed.; Prentice Hall: Englewood Cliff, NJ, USA, 1991; pp. 41-96. 